\title{
Nalmefene in the treatment of pathological gambling: multicentre, double-blind, placebo-controlled study
}

Jon E. Grant, Brian L. Odlaug, Marc N. Potenza, Eric Hollander and Suck Won Kim

\section{Summary}

Pathological gambling is a disabling disorder experienced by about $1 \%$ of adults. We randomised 233 participants $(41.6 \%$ women) 1:1:1 to nalmefene (20 or $40 \mathrm{mg}$ ) or placebo. In analyses performed using an intention-to-treat (ITT) population, nalmefene failed to show statistically significant differences from placebo on primary and secondary outcomes. Post hoc analyses of only participants who received a full titration of the medication for at least 1 week demonstrated that nalmefene $40 \mathrm{mg} /$ day resulted in significantly greater reductions on the primary outcome measure. These findings suggest that medication dosing may be an important consideration in achieving symptom control.

\section{Declaration of interest}

This study was funded by Somaxon Pharmaceuticals, and M.N.P. reports financial interests in Somaxon Pharmaceuticals; he consults for and has been an advisor to Boehringer Ingelheim; has received research support from the National Institutes of Health, Veteran's Administration, Mohegan Sun Casino, National Center for Responsible Gaming (NCRG) and Forest Pharmaceuticals. J.E.G. has received research grants from NIMH, NIDA, NCRG, Forest Pharmaceuticals and GlaxoSmithKline. E.H. has received research grants from Somaxon, Solvay and Abbott.
Pathological gambling is a psychiatric disorder characterised by persistent and recurrent maladaptive patterns of gambling behaviour. Given their efficacy in the treatment of alcohol and opiate dependence, ${ }^{1,2}$ the opioid receptor antagonists naltrexone and nalmefene have been examined in the treatment of pathological gambling. ${ }^{3,4}$ A study of naltrexone $(n=83)$ found that it had greater efficacy than placebo (mean effective dose: $188 \mathrm{mg} /$ day; drop-out rate: $45.8 \%){ }^{3}$ Nalmefene hydrochloride, a long-acting opioid antagonist, has also shown promise in treating pathological gambling. ${ }^{4}$ In a double-blind, 16-week multicentre trial $(n=207)$, various doses of nalmefene $(25,50,1000 \mathrm{mg} /$ day $)$ showed similar efficacy, but premature discontinuation was common (drop-out rate: 66\%). ${ }^{4}$ Based on the encouraging findings from an initial study, the purpose of the present study was to determine whether nalmefene would be safe and effective in the treatment of pathological gambling in a second confirmatory study using different doses. We hypothesised that nalmefene would reduce gambling symptoms and be well tolerated. This study was registered at www.clinicaltrials.gov under number NCT00132119.

\section{Method}

Men and women aged 18-70 years with a primary diagnosis of pathological gambling were recruited by newspaper advertisements. A minimum score of $\geqslant 21$ on the Yale-Brown Obsessive Compulsive Scale Modified for Pathological Gambling (PG-YBOCS), ${ }^{5}$ a minimum score of $\geqslant 5$ on at least one item of the Sheehan Disability Scale (SDS) ${ }^{6}$ and gambling within the 1 month prior to enrolment were required. Exclusion criteria included: current Axis I disorders; current treatment for pathological gambling; and an unstable medical condition. The research was conducted at 25 out-patient centres in the USA. Each treatment centre's institutional review board approved the study and the informed consent. After complete description of the study, participants provided written informed consent.

After screening, eligible participants entered a single-blind, placebo lead-in for 1 week. Those who maintained a PG-YBOCS $\geqslant 15$ after the single-blind phase were randomised (in block sizes of eight, using computer-generated randomisation with no clinical information) to: nalmefene 20 or $40 \mathrm{mg} /$ day; or placebo. Treatment was initiated at $5 \mathrm{mg} /$ day nalmefene or placebo equivalent during the first week of the double-blind phase, and increased to $20 \mathrm{mg} /$ day for the second week. Starting at the third week of this phase, participants randomised to nalmefene $40 \mathrm{mg} /$ day were started on $40 \mathrm{mg} /$ day.

The primary outcome measure was the PG-YBOCS total score. Each PG-YBOCS subscale was evaluated as a secondary efficacy measure. 'Response' rates were based on the total score ${ }^{7}$ (defined as a decrease of $\geqslant 35 \%$ PG-YBOCS at the last evaluation). Secondary outcome measures were the Gambling Symptom Assessment Scale $(\mathrm{G}-\mathrm{SAS})^{8}$ and the Sheehan Disability Scale (SDS) ${ }^{6}$ Safety and laboratory assessments were documented at each visit.

To detect a treatment effect, a sample of 225 will yield $80 \%$ in at least one active arm $v$. placebo. Dunnett simulations were run for the 3-arm trial with each active arm given a standardised treatment effect of 0.5. All randomised participants were included in the analyses of baseline demographics and safety using an intention-to-treat (ITT) principle. Post hoc analyses included only individuals who had attained the full 20 or $40 \mathrm{mg} / \mathrm{day}$ dose for at least 1 week. All tests of hypotheses were performed using a two-sided significance level of 0.05 . The statistical model for the primary variable was a linear mixed model for longitudinal data. ${ }^{9}$ All statistical analyses were carried out using $\mathrm{R}$ for Windows ( $\mathrm{R}$ Foundation, Vienna, Austria; http://www.r-project.org).

\section{Results}

A total of 233 participants (97 (41.6\%) women; mean age 46.5 years, s.d. $=12.0$ ) were randomly assigned to nalmefene $20 \mathrm{mg} / \mathrm{day}$ $(n=77), 40 \mathrm{mg} /$ day $(n=82)$ or placebo $(n=74)$. Pre-planned statistical tests revealed no statistically significant imbalances regarding age, gender, employment, living status or PG-YBOCS total scores among treatment groups. Premature discontinuation was moderate $(46 \%, 107 / 233)$ (Fig. DS1).

In the ITT analyses, $46.8 \%$ of participants assigned to $20 \mathrm{mg} /$ day, $56.1 \%$ of those assigned to $40 \mathrm{mg} /$ day and $59.5 \%$ of those taking placebo were 'responders' $\left(\chi^{2}=2.661\right.$, d.f. $\left.=2, P=0.264\right)$. On the PG-YBOCS, a between-group analysis failed to demonstrate superiority for nalmefene at either dose $(F=1.741$, d.f. $=2$, $P=0.178)$. Neither dose of nalmefene demonstrated statistical differences from placebo on any secondary outcome measure. 
In the post hoc analysis, using only individuals who had a full titration of the assigned doses for at least 1 week ( $73 \%$ of participants assigned to nalmefene), the parameter estimates from the analysis of the mean PG-YBOCS total scores, and all secondary measures, are shown in Table 1. There were statistically significant differences between the $40 \mathrm{mg} /$ day group and placebo on the PG-YBOCS total score at study end-point $(t=-1.96$, d.f. $=14, P=0.035)$.

The incidence and severity of adverse experiences in the nalmefene group were consistent with prior studies and no unusual experiences were reported. Mean values of liver function tests remained within the normal range during the course of the study.

\section{Discussion}

The trial failed to find that either nalmefene 40 or $20 \mathrm{mg} /$ day were superior to placebo in the treatment of pathological gambling. The ITT findings, however, included 43 participants (27\% of the 159 initially assigned to nalmefene) who dropped out before receiving the target dose of nalmefene (20 or $40 \mathrm{mg} /$ day). In the post hoc analysis, which included only individuals who received at least 1 week of target nalmefene dosing, nalmefene $40 \mathrm{mg} /$ day demonstrated statistical advantage over placebo on the main outcome measure of gambling symptoms and specifically the urges associated with pathological gambling. This post hoc finding is consistent with the previous study that found that 25,50 or $100 \mathrm{mg}$ of nalmefene reduced pathological gambling symptoms more than placebo. ${ }^{4}$ The discrepancy between the efficacy outcomes of these two analyses suggests that proper dosing of nalmefene appears to be associated with symptom improvement.

Our post hoc analyses further support the hypothesis that pharmacological manipulation of the opiate system may target core symptoms of pathological gambling. ${ }^{10}$ The efficacy of opioid antagonists in the treatment of addictive disorders, including pathological gambling, has been proposed to involve opioidergic modulation of mesolimbic dopamine circuitry. Further work into defining the precise manner in which opioid antagonists mediate

\begin{tabular}{|c|c|c|c|c|c|}
\hline $\begin{array}{l}\text { Efficacy } \\
\text { measure }\end{array}$ & $\begin{array}{c}\text { At } \\
\text { base- } \\
\text { line }\end{array}$ & $\begin{array}{c}\text { At } \\
\text { week } \\
3^{a}\end{array}$ & $\begin{array}{l}\text { Weekly } \\
\text { decrease } \\
\text { in } \text { LOG }^{\mathrm{b}}\end{array}$ & $\begin{array}{l}\text { Effect } \\
\text { size, } t \text {-test } \\
\quad \text { with } \\
\text { d.f. }=14\end{array}$ & $\begin{array}{l}\text { At final } \\
\text { visit, } \\
95 \% \mathrm{Cl}\end{array}$ \\
\hline \multicolumn{6}{|l|}{ PG-YBOCS, total score } \\
\hline Placebo $(n=71)$ & 21.98 & 16.53 & -6.83 & & $11.29-12.26$ \\
\hline $20 \mathrm{mg} /$ day $(n=59)$ & 21.17 & 16.18 & -6.26 & 0.49 & $11.34-12.30$ \\
\hline $40 \mathrm{mg} /$ day $(n=57)$ & 20.75 & 13.22 & -9.13 & $-1.96 *$ & $6.06-7.21$ \\
\hline \multicolumn{6}{|l|}{$\begin{array}{l}\text { PG-YBOCS, urge/ } \\
\text { thought subscale }\end{array}$} \\
\hline Placebo $(n=71)$ & 11.33 & 8.61 & -3.36 & & $6.01-6.48$ \\
\hline $20 \mathrm{mg} /$ day $(n=59)$ & 10.83 & 8.24 & -3.23 & 0.22 & $5.74-6.22$ \\
\hline $40 \mathrm{mg} /$ day $(n=57)$ & 11.09 & 7.48 & -4.62 & $-2.11^{\star}$ & $4.04-4.61$ \\
\hline \multicolumn{6}{|l|}{$\begin{array}{l}\text { PG-YBOCS, behaviour } \\
\text { subscale }\end{array}$} \\
\hline Placebo $(n=71)$ & 10.66 & 7.90 & -3.51 & & $5.24-5.74$ \\
\hline $20 \mathrm{mg} /$ day $(n=59)$ & 10.35 & 7.93 & -3.05 & 0.72 & $5.58-6.07$ \\
\hline $40 \mathrm{mg} /$ day $(n=57)$ & 10.41 & 6.97 & -4.50 & -1.51 & $3.65-4.24$ \\
\hline \multicolumn{6}{|l|}{ GSAS } \\
\hline Placebo $(n=71)$ & 30.12 & 24.69 & -6.84 & & $19.32-20.55$ \\
\hline $20 \mathrm{mg} /$ day $(n=59)$ & 28.89 & 24.00 & -6.14 & 0.52 & $19.11-20.32$ \\
\hline $40 \mathrm{mg} /$ day $(n=57)$ & 28.24 & 21.56 & -8.60 & -1.28 & $14.98-16.44$ \\
\hline \multicolumn{6}{|c|}{$\begin{array}{l}\text { PG-YBOCS, Pathological Gambling Modification of the Yale-Brown Obsessive } \\
\text { Compulsive Scale; GSAS, Gambling Symptom Assessment Scale. } \\
\text { a. Week } 3 \text { is when both nalmefene groups received full titration of dose for at least } \\
1 \text { week in the post hoc analyses. } \\
\text { b. Natural log of visit number used in analysis. This is a non-linear function of change } \\
\text { in efficacy measure over time. } \\
\star P<0.05 \text {. }\end{array}$} \\
\hline
\end{tabular}

their beneficial effects, and which people will benefit most from these drugs, could enhance treatment for pathological gambling and other impulse control disorders. The high drop out from this study raises the question of whether nalmefene is pharmacologically aversive in this group. This seems unlikely given that none of the participants' gambling symptoms worsened during the study, and the drop-out rate is consistent with that reported in pathological gambling studies of cognitive-behavioural therapy (up to 52\%). ${ }^{11}$

This study has limitations. First, nalmefene only demonstrated superiority in the post hoc analysis of a subset of participants. Use of this more selective subsample for the efficacy analysis makes it very likely that efficacy was overestimated. Given the modest efficacy demonstrated even with this manipulation of the data, it seems likely that nalmefene in either dose failed to separate from placebo using a more conventional ITT approach. Second, pathological gambling may require long-term therapy. Third, the large number of trial centres $(n=25)$ may have affected the trial outcome. Finally, we enrolled only individuals without current comorbidities who were seeking pharmacological treatment, not psychotherapy.

Our post hoc analyses suggest that nalmefene $40 \mathrm{mg} /$ day may be effective in the acute treatment of pathological gambling. As effective treatments for pathological gambling emerge, it becomes increasingly important that physicians and mental healthcare providers screen for it in order to provide timely treatment.

\section{Funding}

This study was funded by Somaxon Pharmaceuticals.

Jon E. Grant, JD, MD, MPH, Brian L. Odlaug, BA, Department of Psychiatry, Jon E. Grant, JD, MD, MPH, Brian L. Odlaug, BA, Department of Psychiatry,
University of Minnesota School of Medicine, Minneapolis, USA; Marc N. Potenza, MD, PhD, Department of Psychiatry, Yale University Medical School, New Haven, Connecticut; Eric Hollander, MD, Department of Psychiatry, Albert Einstein College of Medicine, New York; Suck Won Kim, MD, Department of Psychiatry, University of Minnesota School of Medicine, Minneapolis, USA

Correspondence: Dr Jon E. Grant, Department of Psychiatry, University of Minnesota Medical School, 2450 Riverside Avenue, Minneapolis, MN 55454 USA. Email: grant045@umn.edu

First received 23 Jan 2010, final revision 27 Apr 2010, accepted 19 May 2010

\section{References}

1 Martin WR, Jasinski D, Mansky P. Naltrexone, an antagonist for the treatment of heroin dependence. Arch Gen Psychiatry 1973; 23: 784-89.

2 Volpicelli JR, Alterman Al, Hayashida M, O'Brien CP. Naltrexone in the treatment of alcohol dependence. Arch Gen Psychiatry 1992; 49: 876-80.

3 Kim SW, Grant JE, Adson DE, Shin YC. Double-blind naltrexone and placebo comparison study in the treatment of pathological gambling. Biol Psychiatry 2001; 49: 914-21.

4 Grant JE, Potenza MN, Hollander E, Cunningham-Williams R, Nurminen T, Smits G, et al. Multicenter investigation of the opioid antagonist nalmefene in the treatment of pathological gambling. Am J Psychiatry 2006; 163: 303-12.

5 Pallanti S, DeCaria CM, Grant JE, Urpe M, Hollander E. Reliability and validity of the pathological gambling adaptation of the Yale-Brown ObsessiveCompulsive Scale (PG-YBOCS). J Gambl Stud 2005; 21: 431-43.

6 Sheehan DV. The Anxiety Disease. Scribners, 1983.

7 Hollander E, Pallanti S, Allen A, Sood E, Baldini Rossi N. Does sustainedrelease lithium reduce impulsive gambling and affective instability versus placebo in pathological gamblers with bipolar spectrum disorders? Am J Psychiatry 2005; 162: 137-45.

8 Kim SW, Grant JE, Potenza MN, Blanco C, Hollander E. The Gambling Symptom Assessment Scale (G-SAS): a reliability and validity study. Psychiatry Res 2009; 166: 76-84.

9 Laird NM, Ware JH. Random-effects models for longitudinal data. Biometrics 1982; 38: 963-74.

$10 \mathrm{Kim}$ SW. Opioid antagonists in the treatment of impulse-control disorders. J Clin Psychiatry 1998; 59: 159-64.

11 Sylvain C, Ladouceur R, Boisvert JM. Cognitive and behavioral treatment of pathological gambling: a controlled study. J Consult Clin Psychol 1997; 65: 727-32. 